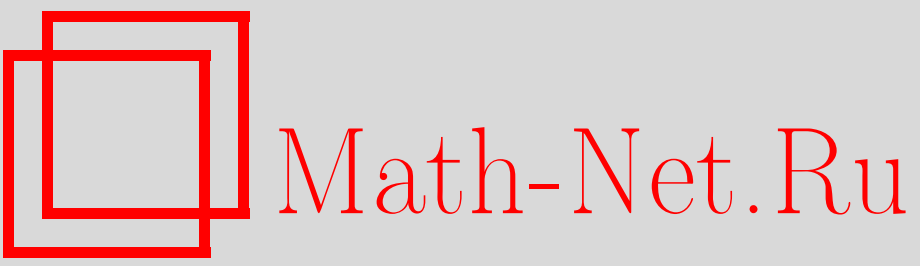

Е. Б. Дынкин, Супердиффузии и положительные решения нелинейных дифференциальных уравнений с частными производными, УМН, 2004, том 59, выпуск $1,145-156$

DOI: https://doi.org/10.4213/rm705

Использование Общероссийского математического портала Math-Net.Ru подразумевает, что вы прочитали и согласны с пользовательским соглашением

http://www.mathnet.ru/rus/agreement

Параметры загрузки:

IP: 3.89.197.203

26 апреля 2023 г., 14:10:42 


\title{
СУПЕР ДИФФУЗИИ И ПОЛОЖИТЕЛЬНЫЕ РЕШЕНИЯ НЕЛИНЕЙНЫХ ДИФФЕРЕНЦИАЛЬНЫХ УРАВНЕНИЙ С ЧАСТНЫМИ ПРОИЗВОДНЫМИ
}

\author{
Е. Б. Дынкин
}

\begin{abstract}
Используя суперброуновское движение, все положительные решения нелинейного дифференциального уравнения $\Delta u=u^{\alpha}$ с $1<\alpha \leqslant 2$ в ограниченной гладкой области $E$ характеризуются их (тонким) следом на границе. Этим решается поставленная несколько лет назад автором задача. Решение для специального случая $\alpha=2$ было дано Б. Мселати в 2002 году.

Библиографиия: 18 названий.
\end{abstract}

\section{СОДЕРЖАНИЕ}

$\S 1$. Введение

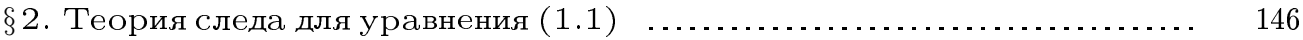

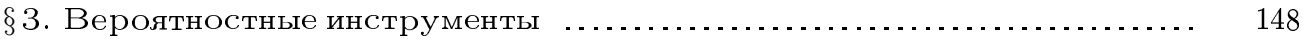

$\S 4$. Соотношения между супердиффузиями и условными дифффузиями $\ldots . . \quad 151$

$\S 5$. Все решения уравнения (1.2) $\sigma$-умеренны ...................... 152

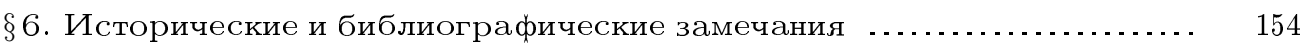

Список литературы ............................................... 155

\section{$\S$ 1. Введение}

1.1. Мы рассматриваем полулинейное дифференщиальное уравнение с частными производньпи

$$
L u=\psi(u) \text { в } E,
$$

где $L$ - эллиптический дифференциальный оператор второго порядка, $E$ - ограниченная гладкая ${ }^{1}$ область в $\mathbb{R}^{d}$ и $\psi$ - непрерывно дифференцируемая положительная функция. Наша цель - описать множество $\mathscr{U}$ всех положительных решений этого уравнения.

Исследование частично поддержано грантом NSF DMS-0204237.

${ }^{1}$ Мы называем гладкими открытые множества класса $C^{2, \lambda}$, если другой класс не указан явно. 
Мы назьваем элемент $u$ множества $\mathscr{U}$ умеренным, если $u \leqslant h$ для некоторого $h$, удовлетворяющего условию $L h=0$ в $E$. Элемент $u$ назьвается $\sigma$-умеренным, если он является пределом возрастаюшей последовательности умеренных решений. При весьма мягких условиях на функцию $\psi$ мы определяем для любой функции $u \in \mathscr{U}$ ее след на границе. Этот след - пара $(\Gamma, \nu)$, где $\Gamma$ - подмножество $\partial E$, а $\nu-\sigma$-конечная мера на $\partial E \backslash \Gamma$. (Точка $y$ принадлежит $\Gamma$, если $\psi^{\prime}(u)$ достаточно быстро стремится к бесконечности при $x \rightarrow y$.) В [7] были описаны все возможные значения следа и было установлено взаимно однозначное соответствие между этими значениями и $\sigma$-умеренными решениями. Возникает естественньй вопрос: все ли решения являются $\sigma$-умеренным.м $?^{2}$ В случае уравнения $\Delta u=u^{2}$ в области класса $C^{4}$ положительный ответ на этот вопрос был дан Б. Мселати в [18]. Однако его основной инструмент - броуновская змея - неприменим к более общим уравнениям.

В [4], [8], [9] и [12] Е. Б. Дынкин и С. Е. Кузнецов изучили уравнение

$$
\Delta u=u^{\alpha} \text { при } 1<\alpha \leqslant 2,
$$

используя супердиффузию вместо броуновской змеи. Бо́льшая часть рассуждений Мселати была перенесена на этот случай. Однако критический пробел оставался незаполненным, пока он не был закрыт в августе 2003 года в [5] с помошю нового неравенства для супердиффузии (см. (5.1)).

1.2. В $\S 2$ мы излагаем теорию следа для уравнения (1.1), развитую в [11] и [7]. Мы отложим до 33 обсуждение связей между уравнением (1.1) и супердифффузией. Вначале эта связь играла решающую роль (см. [11]). Позже теория была распространена на случай уравнений, не соответствуюших никакой супердиффузии (включая уравнение (1.2) с $\alpha>2$ ). В $\S \S 3,4$ мы также готовим вероятностные средства для $\S 5$, где мы даем набросок доказательства того, что все решения уравнения (1.2) $\sigma$-умеренны. Заключительньй $\S 6$ содержит исторические и библиографические замечания.

\section{§ 2. Теория следа для уравнения (1.1)}

2.1. Наша теория приложима к широкому классу непрерьвно дифференцируемых функций $\psi$, удовлетворяюших условиям $\psi(0)=0$ и $\psi(u)>0$ при $u>0 .{ }^{3}$ Bсе результаты остаются справедливьми (с заменой $\partial E$ на границу Мартина) для любой области $E$ и, более обшим образом, для любых дифференщируемых многообразий.

2.2. Умеренные и $\sigma$-умеренные решения. Нашей исходной точкой является представление положительных решений линейного уравнения

$$
L h=0 \quad \text { в } E
$$

интегралами Пуассона. Если $k(x, y)$ - ядро Пуассона для $L$ в $E$, то формула

$$
h_{\nu}(x)=\int_{\partial E} k(x, y) \nu(d y)
$$

\footnotetext{
2Этот вопрос был задан как ключевая нерешенная задача в эпилоге книги [3].

${ }^{3}$ Она также применима к функциям $\psi(x, u)$, зависящим от $x \in E$. См. главу 11 в [3].
} 
устанавливает взаимно однозначное соответствие между множеством $\mathscr{M}(\partial E)$ всех конечных мер $\nu$ на $\partial E$ и множеством $\mathscr{H}$ всех положительных решений (2.1). (Мы называем решения уравнения (2.1) гармоническими функциями.)

Существует взаимно однозначное соответствие между множеством $\mathscr{U}_{1}$ всех умеренных решений и некоторым подмножеством $\mathscr{H}_{1}$ семейства $\mathscr{H}$, а именно, $h \in \mathscr{H}_{1}$ - минимальная гармоническая функция, мажорирующая $u \in \mathscr{U}_{1}$, и $u$ - максимальное решение, мажорируемое $h$. Положим $\nu \in \mathscr{N}_{1}$, если $h_{\nu} \in \mathscr{H}_{1}$. Обозначим через $u_{\nu}$ элемент $\mathscr{U}$, соответствуюший $h_{\nu}$.

Индексирование умеренных решений мерами $\nu \in \mathcal{N}_{1}$ можно продолжить следуюшим образом на $\sigma$-умеренные решения: если $\nu_{n} \in \mathcal{N}_{1}, \nu_{n} \uparrow \nu$ и $u_{\nu_{n}} \uparrow u$, то мы полагаем $\nu \in \mathscr{N}_{0}$ и $u=u_{\nu}$.

2.3. Решеточная структура в $E$. Положим $u \leqslant v$, если $u(x) \leqslant v(x)$ для всех $x \in E$. Это определяет частичньй порядок в $\mathscr{U}$. Для любого $\widetilde{\mathscr{U}} \subset \mathscr{U}$ сушествует единственньй элемент $u$ в $\mathscr{U}$ со следующими свойствами:

(а) $u \geqslant v$ для любого $v \in \mathscr{U}$;

(б) если $\widetilde{u} \in \mathscr{U}$ удовлетворяет условию (а), то $u \leqslant \widetilde{u}$.

Обозначим этот элемент через $\operatorname{Sup} \widetilde{U}$.

Для любых $u, v \in \mathscr{U}$ положим $u \oplus v=\operatorname{Sup} W$, где $W$ - множество всех таких элементов $w \in \mathscr{U}$, что $w \leqslant u+v$. Заметим, что $u \oplus v$-умеренньй элемент, если $u$ и $v$ умеренные, и он $\sigma$-умерен, если $u$ и $v$ являются $\sigma$-умеренными элементами.

Вообще говоря, Sup $\widetilde{\mathscr{U}}$ не совпадает с поточечньм супремумом (который не обязан принадлежать $\mathscr{U}$ ). Однако эти функции совпадают, если множество $\widetilde{\mathscr{U}}$ замкнуто относительно операции $\oplus$. Более того, в этом случае существует такое $u_{n} \in \widetilde{\mathscr{U}}$, что $u_{n}(x) \uparrow u(x)$ для всех $x \in E$. Следовательно, если множество $\widetilde{\mathscr{U}}$ замкнуто относительно операции $\oplus$ и состоит из умеренных решений, то $\operatorname{Sup} \widetilde{U}$ является $\sigma$-умеренной функцией. В частности, любому борелевскому подмножеству Г в $\partial E$ соответствует $\sigma$-умеренное решение

$$
u_{\Gamma}=\operatorname{Sup}\left\{u_{\nu}: \nu \in \mathscr{N}_{1}, \nu \text { сосредоточена на } \Gamma\right\}
$$

Мы сейчас свяжем с Г еше одно решение $w_{\Gamma}$. Во-первых, определим $w_{K}$ для замкнутого множества $K$ формулой

$$
w_{K}=\operatorname{Sup}\{u \in \mathscr{U}: u=0 \text { на } \partial E \backslash K\}
$$

Для любого борелевского подмножества Г в $\partial E$ положим

$$
w_{\Gamma}=\operatorname{Sup}\left\{w_{K}: K \text { - замкнутое множество, } K \subset \Gamma\right\} \text {. }
$$

Доказательство равенства $u_{\Gamma}=w_{\Gamma}$ было важной частью программы, кратко описанной в [3].

2.4. Сингулярные точки решения $u$. Рассмотрим классические решения уравнения (1.1), которые дважды непрерывно дифференцируемы в $E$. Однако они могут 
стремиться к бесконечности при $x \rightarrow y \in \partial E$. Мы будем говорить, что $y-c u н г y$ лярная точка $u$, если она является точкой быстрого роста $\psi^{\prime}(u)$. (Особая роль $\psi^{\prime}(u)$ связана с тем фактом, что касательное пространство к $\mathscr{U}$ в точке $u$ описьвается уравнением $L v=\psi^{\prime}(u) v$.)

Быстрый рост положительной непрерьвной функции $a(x)$ может быть определен как аналитически, так и вероятностно. Аналитическое определение использует ядро Пуассона $k_{a}(x, y)$ оператора $L u-a u: y \in \partial E$ является точкой быстрого роста для $a$, если $k_{a}(x, y)=0$ для всех $x \in E$. Более прозрачное вероятностное определение дано в $\S 3$.

Мы будем говорить, что борелевское подмножество Г в $\partial E$ является $f$-замкнутыл если Г содержит все сингулярные точки решения $u_{\Gamma}$, определенного формулой (2.3).

2.5. Определение и свойства следа. След $u \in \mathscr{U}$ (который мы обозначим через $\operatorname{Tr}(u))$ определяется как пара $(\Gamma, \nu)$, где $\Gamma$ - множество всех сингулярных точек $u$ и $\nu$-мера на $\partial E \backslash \Gamma$, заданная формулой

$$
\nu(B)=\sup \left\{\mu(B): \mu \in \mathscr{N}_{1}, \mu(\Gamma)=0, u_{\mu} \leqslant u\right\}
$$

Имеем

$$
u_{\nu}=\operatorname{Sup}\left\{u_{\mu} \text { умеренно, } u_{\mu} \leqslant u, \mu(\Gamma)=0\right\} \text {, }
$$

и поэтому $u_{\nu}$ является $\sigma$-умеренным.

След любого решения $u$ имеет следующие свойства.

2.5.А. $\Gamma$ - борелевское $f$-замкнутое множество; $\nu$ - такая $\sigma$-конечная мера класса $\mathscr{N}_{0}$, что $\nu(\Gamma)=0$ и все сингулярные точки $u_{\nu}$ принадлежат $\Gamma$.

2.5.Б. Если $\operatorname{Tr}(u)=(\Gamma, \nu)$, то

$$
u \geqslant u_{\Gamma} \oplus u_{\nu}
$$

Кроме того, $u_{\Gamma} \oplus u_{\nu}$ - максимальное $\sigma$-умеренное решение, мажорируемое $u$.

2.5.В. Если $(\Gamma, \nu)$ удовлетворяет условию 2.5.А, то $\operatorname{Tr}\left(u_{\Gamma} \oplus u_{\nu}\right)=\left(\Gamma^{\prime}, \nu\right)$, и симметрическая разность между $Г$ и $\Gamma^{\prime}$ не заряжена никакой мерой $\mu \in \mathscr{N}_{1}$. Кроме того, $u_{\Gamma} \oplus u_{\nu}$ является минимальным решением с этим свойством, и при этом единственным $\sigma$-умеренным решением.

\section{§ 3. Вероятностные инструменты}

3.1. Диффузия. При некоторых мягких предположениях относительно коэффициентов $L$ сушествует непрерьвный марковский процесс $\xi=\left(\xi_{t}, \Pi_{x}\right)$ в $\mathbb{R}^{d}$, переходная плотность которого является фундаментальным решением параболического уравнения $\partial u / \partial t=L u$. Мы назьваем этот процесс диффузией. Для любого открытого множества $D$ обозначим через $\tau_{D}$ время первого выхода $\xi$ из $D$.

3.2. Условная диффузия. Пусть $\xi$ - диффузия в области $E$ с переходной функцией $p_{t}(x, d y)$, и пусть $h \in \mathscr{H}$. Тогда

$$
p_{t}^{h}(x, d y)=\frac{1}{h(x)} p_{t}(x, d y) h(y)
$$


- переходная функция непрерьвного марковского процесса $\left(\xi_{t}, \widehat{\Pi}_{x}^{h}\right)$ в $E$, который называется $h$-преобразованием $\xi$. Мы предпочитаем иметь дело с мерами $\Pi_{x}^{h}=h(x) \widehat{\Pi}_{x}^{h}$, которые линейно зависят от $h$. Положим $\Pi_{x}^{\nu}=\Pi_{x}^{h_{\nu}}$ и $\widehat{\Pi}_{x}^{y}=\widehat{\Pi}_{x}^{\delta_{y}}$, где $\delta_{y}$ - единичная масса в точке $y$. Процесс $\left(\xi_{t}, \widehat{\Pi}_{x}^{y}\right)$ можно интерпретировать как диффузию, начинаюшуюся в $x \in E$ и обусловленную выходом из $E$ в точке $y \in \partial E$.

Вероятностное определение быстрого роста положительной непрерывной функции $a(x), x \in E$, может быть сформулировано в терминах условной диффузии следующим образом: $y \in \partial E$ есть точка быстрого роста для $a$, если

$$
\widehat{\Pi}_{x}^{y}\left[\int_{0}^{\tau_{E}} a\left(\xi_{t}\right) d t=\infty\right]=1 \quad \text { для любого } x \in E .
$$

3.3. Супердиффузия. Обозначим через $\mathscr{M}(S)$ множество всех конечных мер и через $\mathscr{P}(S)$ множество всех вероятностных мер на пространстве $S$, снабженном семейством измеримых множеств. Пусть $\mathscr{B}(E)$ означает множество всех положительных борелевских функций на $E \subset \mathbb{R}^{d}$. Обозначим через $\langle u, \mu\rangle$ интеграл от $u$ по мере $\mu$ и используем обозначение $P\{A, Y\}$ для $\int_{A} Y d P$.

Предположим, что любому открытому множеству $D$ и любой мере $\mu \in \mathscr{M}\left(\mathbb{R}^{d}\right)$ сопоставлена такая случайная мера $\left(X_{D}, P_{\mu}\right)$ на $\mathbb{R}^{d}$, что

$$
P_{\mu} e^{-\left\langle f, X_{D}\right\rangle}=e^{-\langle u, \mu\rangle}
$$

для любой функции $f \in \mathscr{B}\left(\mathbb{R}^{d}\right)$, где $u$ удовлетворяет уравнению

$$
u(x)+\Pi_{x} \int_{0}^{\tau_{D}} \psi\left[u\left(\xi_{t}\right)\right] d t=\Pi_{x} f\left(\xi_{\tau_{D}}\right)
$$

Мы назовем $X=\left(X_{D}, P_{\mu}\right)$ супердиффузией. (Эвристически, мы имеем здесь модель случайной эволюции облака частиц, и $X_{D}$ является распределением масс на $\partial D$, если любая частица замораживается в момент своего первого выхода из $D .{ }^{4}$ ) Существование супердиффузии доказано для вьпуклого класса функций $\psi$, содержашего функции $\psi(u)=u^{\alpha}$ при $1<\alpha \leqslant 2$.

Обозначим через $\mathscr{F} \subset D \quad \sigma$-алгебру, порожденную $X_{D^{\prime}}, D^{\prime} \subset D$, а через $\mathscr{F}_{\supset D}-$ $\sigma$-алгебру, порожденную $X_{D^{\prime \prime}}, D^{\prime \prime} \supset D$.

В теории супердиффузии и ее приложениях важную роль играют следуюшие два свойства:

Марковость. Если $Y \geqslant 0$ является $\mathscr{F} \subset D^{-и з м е р и м ы м ~ и ~} Z \geqslant 0$ является $\mathscr{F} \supset D^{-и з-}$ меримым, то

$$
P_{\mu}(Y Z)=P_{\mu}\left(Y P_{X_{D}} Z\right)
$$

Абсолютная непрерывность. При любых $x_{1}, x_{2} \in D \quad P_{x_{1}}$ абсолютно непрерьвна относительно $P_{x_{2}}$ на $\sigma$-алгебре $\mathscr{F} \supset D$.

\footnotetext{
${ }^{4}$ Процесс $\xi$ описьвает движение каждой частицы и $\psi$ характеризует механизм размножения.
} 
3.4. Меры $\mathbb{N}_{x}$. Новый инструмент - семейство мер $\mathbb{N}_{x}, x \in E$ (определенных на том же пространстве $\Omega$, что и меры $\left.P_{\mu}\right),-$ был введен в [9]. Нас мотивировала роль, которую аналог этих мер играет в теории Ле Галя броуновских змей.

Обозначим через $\mathscr{Z}_{x}$ класс всех функций вида

$$
Z=\sum_{1}^{n}\left\langle f_{i}, X_{O_{i}}\right\rangle
$$

где $O_{1}, \ldots, O_{n}$ - конечное семейство окрестностей $x$ и $f_{1}, \ldots, f_{n} \in \mathscr{B}\left(\mathbb{R}^{d}\right)$. По теореме 1.1 в [8] сушествует единственное семейство мер $\mathbb{N}_{x}$ со следующими свойствами.

(i) Для любого $Z \in \mathscr{Z}_{x}$ имеем

$$
\mathbb{N}_{x}\left(1-e^{-Z}\right)=-\ln P_{x} e^{-Z}
$$

(ii) Если $\bar{\Omega}$ - пересечение множеств $\left\{X_{O}=0\right\}$ по всем окрестностям $x$, то $\mathbb{N}_{x}(\bar{\Omega})=0$.

3.5. Стохастические граничные значения и ранг. Предположим, что $u \in$ $\mathscr{B}(E)$. Случайная величина $Z$ назьвается стохастическим граничным значением $u$ (мы пишем $Z=\operatorname{SBV}(u)$ ), если

$$
Z=\lim \left\langle u, X_{D_{n}}\right\rangle \quad P_{x} \text {-п.н. и } \mathbb{N}_{x} \text {-П.н. }
$$

для всех $x \in E$ и для любой последовательности $D_{n}$, исчерпьвающей $E .^{5}$

Стохастические граничные значения существуют для всех $h \in \mathscr{H}$ и всех $u \in \mathscr{U}$, и если $Z_{u}=\operatorname{SBV}(u)$, то

$$
u(x)=\mathbb{N}_{x}\left(1-e^{-Z_{u}}\right)=-\ln P_{x} e^{-Z_{u}} .
$$

Для любой области $E$ сушествует случайное замкнутое множество $\mathscr{R}_{E}$ со следуюшими свойствами.

(а) Для любого открытого множества $O \subset E$ и любого $x \in E$ мера $X_{O}$ сосредоточена $\left(P_{x}\right.$-п.н. и $\mathbb{N}_{x}$-П.н.) на $\mathscr{R}_{E}$.

(б) Если (а) вьполняется для случайного замкнутого множества $F$, то для любого $x \in E$ соотношение $\mathscr{R}_{E} \subset F$ вьполняется $P_{x}$-п.н. и $\mathbb{N}_{x}$-п.н.

Назовем $\mathscr{R}_{E}$ рангом $X$ в $E$ и обозначим через $\mathscr{R}$ ранг $X$ в $\mathbb{R}^{d}$.

3.6. Вероятностное представление решения. Положим

$$
Z_{\nu}=\operatorname{SBV}\left(h_{\nu}\right)
$$

Если $\nu \in \mathscr{N}_{1}$, то $Z_{\nu}=\operatorname{SBV}\left(u_{\nu}\right)$ и

$$
u_{\nu}(x)=-\ln P_{x} e^{-Z_{\nu}}=\mathbb{N}_{x}\left(1-e^{-Z_{\nu}}\right) .
$$

\footnotetext{
${ }^{5}$ Мы говорим, что области $D_{n}$ исчерпывают $E$, если $\bar{D}_{n} \subset D_{n+1}$ и если $E$ является объединением всех $D_{n}$.
} 
Теперь решение $w_{\Gamma}$ можно представить формулой

$$
w_{\Gamma}(x)=-\ln P_{x}\left(\mathscr{R}_{E} \cap \Gamma=\varnothing\right)=\mathbb{N}_{x}\left(\mathscr{R}_{E} \cap \Gamma \neq \varnothing\right),
$$

и

$$
\begin{aligned}
\left(w_{\Gamma} \oplus u_{\nu}\right)(x) & =-\ln P_{x}\left\{\mathscr{R}_{E} \cap \Gamma=\varnothing, e^{-Z \nu}\right\} \\
& =\mathbb{N}_{x}\left(\mathscr{R}_{E} \cap \Gamma \neq \varnothing\right)+\mathbb{N}_{x}\left\{\mathscr{R}_{E} \cap \Gamma=\varnothing, 1-e^{-Z_{\nu}}\right\} .
\end{aligned}
$$

\section{$\S 4$. Соотношения между супердиффузиями и условными диффузиями}

Эти соотношения (установленные в [5]) играют ключевую роль в следующем паparрафе. $^{6}$

Положим

$$
\mathscr{E}_{x}(\nu)=\Pi_{x} \int_{0}^{\tau_{E}} \psi\left[h_{\nu}\left(\xi_{t}\right)\right] d t .
$$

Если $\mathscr{E}_{x}(\nu)<\infty$ для некоторого $x \in E$, то $\nu \in \mathscr{N}_{1}$.

Справедливы следующие утверждения.

4.A. Пусть $Z_{\nu}=\operatorname{SBV}(h)$ и $Z_{u}=\operatorname{SBV}(u)$, где $\nu \in \mathscr{N}_{1}$ и $u \in \mathscr{U}$. Тогда

$$
P_{x} Z_{\nu} e^{-Z_{u}}=e^{-u(x)} \Pi_{x}^{\nu} e^{-\Phi(u)}
$$

и

$$
\mathbb{N}_{x} Z_{\nu} e^{-Z_{u}}=\Pi_{x}^{\nu} e^{-\Phi(u)},
$$

где

$$
\Phi(u)=\int_{0}^{\tau_{E}} \psi^{\prime}\left[u\left(\xi_{s}\right)\right] d s .
$$

4.Б. Предположим, что $D$ - гладкое открытое подмножество $E$. Обозначим через $\Lambda$ замькание $\partial D \cap E$ и положим $L=\partial D \backslash \Lambda$ и $D^{*}=D \cup L$. Пусть

$$
\begin{gathered}
w_{\Lambda}(x)=\mathbb{N}_{x}\left\{\mathscr{R}_{E} \cap \Lambda \neq \varnothing\right\}, \\
v_{s}(x)=w_{\Lambda}(x)+\mathbb{N}_{x}\left\{\mathscr{R}_{E} \cap \Lambda=\varnothing, 1-e^{-s Z_{\nu}}\right\} .
\end{gathered}
$$

Если $x \in D$, а $\nu$ - такая конечная мера на $\partial D \cap \partial E$, что $\mathscr{E}_{x}(\nu)<\infty$, то

$$
\begin{gathered}
\mathbb{N}_{x}\left\{\mathscr{R}_{E} \subset D^{*}, Z_{\nu}\right\}=\Pi_{x}^{\nu}\left\{A, e^{-\Phi\left(w_{\Lambda}\right)}\right\} \\
\mathbb{N}_{x}\left\{\mathscr{R}_{E} \subset D^{*}, Z_{\nu} \neq 0\right\}=\int_{0}^{\infty} \Pi_{x}^{\nu}\left\{A, e^{-\Phi\left(v_{s}\right)}\right\} d s,
\end{gathered}
$$

\footnotetext{
${ }^{6}$ Формула (4.2) была доказана ранее в [2]. Аналог равенства (4.3) для броуновской змеи можно найти в [18].
} 
где $\Phi$ определена формулой (4.4) и

$$
A=\left\{\tau_{E}=\tau_{D}\right\}=\left\{\xi_{t} \in D \text { для всех } t<\tau_{E}\right\}
$$

\section{$\S 5$. Все решения уравнения (1.2) $\sigma$-умеренны}

5.1. Пусть $u \in \mathscr{U}$ и $\operatorname{Tr}(u)=(\Gamma, \nu)$. Доказательство того, что $u \sigma$-умеренно, состоит из трех частей:
A. $u \geqslant u_{\Gamma} \oplus u_{\nu}$,
B. $u \leqslant w_{\Gamma} \oplus u_{\nu}$,
C. $u_{\Gamma}=w_{\Gamma}$.

Из А-С следует, что $u=u_{\Gamma} \oplus u_{\nu}=w_{\Gamma} \oplus u_{\nu}$, и поэтому $u$ является $\sigma$-умеренньм решением.

Мы уже сформулировали утверждение А как часть теории следа, охватьвающей общее уравнение (1.1) (см. (2.7)). Часть В была доказана Мселати [18] для уравнения $\Delta u=u^{2}$. В [4] это утверждение было распространено на случай уравнения (1.2).

Доказательство утверждения С можно свести к доказательству того факта, что $w_{K} \leqslant C u_{K}$ для замкнутого множества $K$ (см. [18; предложения 3.0 .1 и 3.0.2]). Чтобы доказать эту оценку, мы используем новое неравенство для супердиффузии и неравенства для $w_{K}$ и $u_{K}$ в терминах пуассоновых емкостей. ${ }^{7}$

(Доказательства утверждений В и С зависят от неравенства (5.6), которое в настоящее время установлено для областей класса $C^{4}$. Если доказать неравенство (5.6) для областей класса $C^{2, \lambda}$, то В и С будут автоматически доказаны для этих областей.)

5.2. Новое неравенство для супердиффузии. Следуюший результат получен в [5] из утверждений 4. А и 4.Б.

Предположим, что $D \subset E$ - ограниченные гладкие открытые множества и $\Lambda, L, D^{*}$ - множества, введенные в 4.Б. Пусть $\nu$ - конечная мера на $\partial D \cap \partial E, x \in E$ и $\mathscr{E}_{x}(\nu)<\infty$. Тогда

$$
\mathbb{N}_{x}\left\{\mathscr{R}_{E} \subset D^{*}=\varnothing, Z_{\nu} \neq 0\right\} \geqslant C(\alpha)\left[\mathbb{N}_{x}\left\{\mathscr{R}_{E} \subset D^{*}, Z_{\nu}\right\}\right]^{\alpha /(\alpha-1)} \mathscr{E}_{x}(\nu)^{-1 /(\alpha-1)}
$$

где $C(\alpha)=(\alpha-1)^{-1} \Gamma(\alpha-1) .^{8}$

5.3. Пуассоновы емкости. Для любого борелевского подмножества $B$ в $\partial E$ положим

$$
\operatorname{Cap}_{x}(B)=\sup _{\nu \in \mathscr{P}(B)} \mathscr{E}_{x}(\nu)^{-1}
$$

Эта формула сопоставляет емкость Шоке каждой точке $x \in E$. Мы также вводим емкость

$$
\operatorname{Cap}(B)=\sup _{\nu \in \mathscr{P}(B)} \mathscr{E}(\nu)^{-1}
$$

\footnotetext{
${ }^{7}$ М. Маркус и Л. Верон анонсировали в [17], что равенство $u_{K}=w_{K}$ может быть доказано при $\alpha>1$ другим, чисто аналитическим способом. Доказателшство еще не опубликовано, но некоторые указания приведены в [17].

${ }^{8} \Gamma$ - гамма-ффункция Эйлера.
} 
где

$$
\mathscr{E}(\nu)=\int_{E} \rho(x) h_{\nu}(x)^{\alpha} d x .
$$

(Здесь $\rho(x)$ означает расстояние от $x$ до гранищы $\partial E$.)

Обозначим через $d(x, K)$ расстояние от $x$ до $K$ и через $\operatorname{diam}(K)$ диаметр $K$. Положим

$$
E_{\kappa}(K)=\{x \in E: d(x, K) \geqslant \kappa \operatorname{diam}(K)\}, \quad \varphi(x, K)=\rho(x) d(x, K)^{-d} .
$$

Следуюшее соотношение между Сар и Сар $x$ было доказано в [8].

Существует такая постоянная ${ }^{9} C_{\kappa}$, что для любого замкнутого множества $K \subset \partial E$ имеем

$$
\operatorname{Cap}(K) \leqslant C_{\kappa} \varphi(x, K) \operatorname{Cap}_{x}(K) \text { для всех } x \in E_{\kappa}(K) \text {. }
$$

5.4. Верхняя граница для $w_{K}$. Предположим, что $d \geqslant 4 .{ }^{10} \mathrm{~B}[12]$ доказано, что сушествует постоянная $C$, удовлетворяюшая условию

$$
w_{K}(x) \leqslant C \varphi(x, K) \operatorname{Cap}(K)^{1 /(\alpha-1)}
$$

для любого $K$ и для любого $x \in E$. Из (5.6) и (5.5) следует, что для любого $\kappa>0$ существует такая постоянная $C_{\kappa}$, что для любого компактного множества $K \subset \partial E$ и любого $x \in E_{\kappa}$ имеем

$$
w_{K}(x) \leqslant C_{\kappa}\left[\varphi(x, K)^{\alpha} \operatorname{Cap}_{x}(K)\right]^{1 /(\alpha-1)} .
$$

5.5. Нижние оценки для $u_{K}$. По определению $u_{K}$ (см. (2.3)), $u_{K} \geqslant u_{\nu}$ для любой меры $\nu \in \mathscr{N}_{1}$, сосредоточенной на $K$. Так как $t \nu \in \mathscr{N}_{1}$ для всех $t>0$, то

$$
u_{K} \geqslant u_{\infty \nu}=\lim _{t \rightarrow \infty} u_{t \nu}
$$

Из формулы (3.7) следует, что $u_{\infty \nu}=\mathbb{N}_{x}\left\{Z_{\nu} \neq 0\right\}$. Следуя Мселати, мы заменим эту функцию меньшей функцией

$$
\mathbb{N}_{x}\left\{\mathscr{R} \in \mathscr{B}_{n}(x, K), Z_{\nu} \neq 0\right\}
$$

где $B_{n}(x, K)=\{z:|x-z|<n d(x, K)\}$. Основное неравенство (5.1) позволяет заменить оценку функции (5.8) оценкой величины $\mathbb{N}_{x}\left\{\mathscr{R} \in \mathscr{B}_{n}(x, K), Z_{\nu}\right\}$. Мы сначала установим следуюшее утверждение.

Сушествуют такие постоянные $n$ и $C_{\kappa}>0$, что

$$
\mathbb{N}_{x}\left\{\mathscr{R} \subset B_{n}(x, K), Z_{\nu}\right\} \geqslant C_{\kappa} \varphi(x, K)
$$

\footnotetext{
${ }^{9} C_{\kappa}=1 \vee \kappa \kappa^{\gamma}$, где $\gamma=0 \vee(d \alpha-d-\alpha-1)$. Если $\alpha<(d+1) /(d-1)$, то $\gamma=0$, и $(5.5)$ вьполняется при $C_{\kappa}=1$ для всех $x \in E$.

${ }^{10}$ Случай $d<4$ может быть изучен отдельно. Здесь мы его не рассматриваем.
} 
для любого замкнутого подмножества $K$ в $\partial E$ и для любых $x \in E_{\kappa}(K)$ и всех $\nu \in$ $\mathscr{P}(K)$ таких, что $\mathscr{E}_{x}(\nu)<\infty$.

При $\alpha=2$ это утверждение следует из леммы 3.2.2 в [18]. Рассуждения Мселати можно модифищировать так, чтобы покрыть случай более общего уравнения (1.2).

Используя (5.1), (5.9) и (5.2), мы можем доказать следующее утверждение.

Сушествуют постоянные $C_{\kappa}>0$ и $n$ со следуюшим свойством: для любого замкнутого $K \subset \partial E$ и любого $x \in E_{\kappa}(K)$ вьполняется неравенство

$$
\mathbb{N}_{x}\left\{\mathscr{R} \subset B_{2 n}(x, K), Z_{\nu} \neq 0\right\} \geqslant C_{\kappa}\left[\varphi(x, K)^{\alpha} \operatorname{Cap}_{x}(K)\right]^{1 /(\alpha-1)}
$$

для некоторой меры $\nu \in \mathscr{P}(K)$, удовлетворяющей условию $\mathscr{E}_{x}(\nu)<\infty$.

5.6. Из (5.7) и (5.10) вытекает следующее утверждение.

Сушествуют такие постоянные $C_{\kappa}>0$ и $n$, что для любого замкнутого множества $K \subset \partial E$ и любого $x \in E_{\kappa}(K)$ вьполняется неравенство

$$
w_{K}(x) \leqslant C_{\kappa} \mathbb{N}_{x}\left\{\mathscr{R}_{E} \subset B_{2 n}(x, K), Z_{\nu} \neq 0\right\}
$$

для некоторой меры $\nu \in \mathscr{P}(K)$, удовлетворяющей условию $\mathscr{E}_{x}(\nu)<\infty$.

Используя рассуждения статьи [18] (с. 93-95), мы получаем отсюда, что $w_{K} \leqslant$ $C u_{K}$.

\section{§. Исторические и библиографические замечания}

След $\operatorname{Tr}(u)$ был введен в [11] и [7] под названием тонкого следа. Было предложено называть вариант следа, которьй рассматривался в литературе ранее, грубым следом. (В монографии [3] грубый след изучается в главе 10, а тонкий след вводится и изучается в главе 11.) В субкритическом случае $1<d<\frac{\alpha+1}{\alpha-1}$ грубый след совпадает с тонким следом и однозначно определяет решение уравнения (1.2). Как показал Ле Галь, это не так в суперкритическом случае $d \geqslant \frac{\alpha+1}{\alpha-1}$.

В пионерской работе [10] А. Гмира и Л. Верон доказали, что в субкритическом случае обобщенная задача Дирихле

$$
\begin{gathered}
\Delta u=u^{\alpha} \quad \text { в } E, \\
u=\mu \text { на } \partial E
\end{gathered}
$$

имеет единственное решение для любой конечной меры $\mu$. (В наших обозначениях это решение есть $u_{\mu}$.)

Программа исследования $\mathscr{U}$ с помощью супердиффузии была инищиирована в [1]. В частности, автор высказал гипотезу, что для любого $\alpha, 1<\alpha \leqslant 2$, и любого $d$ задача (1.2) имеет решение в том и только том случае, когда $\mu(\Gamma)=0$ для всякого Г, не пересекающегося почти наверное с рангом супердиффузии. ${ }^{11}$ (Гипотеза была доказана сначала Ле Галем в случае $\alpha=2$ и затем для всех $1<\alpha \leqslant 2$ Дьнкиным и Кузнецовьгм.)

\footnotetext{
${ }^{11}$ Ограничение $\alpha \leqslant 2$ необходимо, посколку соответствующая супердифффузия существует только в этой области.
} 
Классификация всех положительных решений уравнения $\Delta u=u^{2}$ в единичном диске $E=\left\{x \in \mathbb{R}^{2}:|x|<1\right\}$ была анонсирована Ле Галем в [13]. (Это - тоже субкритический случай.) Этот результат был доказан и распространен на широкий класс гладких плоских областей в [14]. Вместо супердиффузии Ле Галь использовал свой собственное изобретение - процесс со значениями в пространстве путей, так назьваемую броуновскую змею. Он установил взаимно однозначное соответствие между $\mathscr{U}$ и парами $(\Gamma, \nu)$, где $\Gamma$ - замкнутое подмножество $\partial E$, а $\nu$ - радонова мера на $\partial E \backslash \Gamma$.

Дьнкин и Кузнецов [6] распространили результат Ле Галя на случай уравнения $L u=u^{\alpha}, 1<\alpha \leqslant 2$. Они ввели грубый граничный след для решений этого уравнения, описали все возможные значения следа и представили максимальное решение с данньм следом в терминах супердиффузии.

Маркус и Верон [15], [16] исследовали грубый след решений чисто аналитическими средствами. Они распространили теорию на случай $\alpha>2$ и доказали, что грубый след однозначно определяет решение в субкритическом случае.

Теория тонкого следа, развитая в [7], привела к классификации всех $\sigma$-умеренных решений. Диссертация Мселати [18] завершила эту классификацию для уравнения $\Delta u=u^{2}$; там было доказано, что в рассматриваемом случае все решения являются $\sigma$-умеренными. Результаты [5], представленные в $\S 5$, распространяют этот вывод на случай более общего уравнения (1.2).

\section{СПИСОК ЛИТЕРАТУРЫ}

[1] E. B. Dynkin. A probabilistic approach to one class of nonlinear differential equations // Probab. Theory Related Fields. 1991. V. 89. № 1. P. 89-115.

[2] E. B. Dynkin. A new relation between diffusions and superdiffusions with applications to the equation $L u=u^{\alpha} / /$ C. R. Acad. Sci. Paris Sér. I Math. 1997. V. 325. № 4. P. 439-444.

[3] E. B. Dynkin. Diffusions, Superdiffusions and Partial Differential Equations. Providence, RI: Amer. Math. Soc., 2002.

[4] E. B. Dynkin. On upper bounds for positive solutions of semilinear equations // J. Funct. Anal. (to appear).

[5] E. B. Dynkin. New relations between diffusions and superdiffusions and their applications to differential equations // Math. Res. Lett., submitted.

[6] E. B. Dynkin, S. E. Kuznetsov. Trace on the boundary for solutions of nonlinear differential equations // Trans. Amer. Math. Soc. 1998. V. 350. № 11. P. 4499-4519.

[7] E. B. Dynkin, S. E. Kuznetsov. Fine topology and fine trace on the boundary associated with a class of semilinear differential equations // Comm. Pure Appl. Math. 1998. V. 51. № 8. P. 897-936.

[8] E. B. Dynkin, S. E. Kuznetsov. Poisson capacities // Math. Res. Lett. 2003. V. 10. № 1. P. $85-95$.

[9] E. B. Dynkin, S.E. Kuznetsov. NN-measures for branching exit Markov systems and their applications to differential equations // Probab. Theory Related Fields, submitted.

[10] A. Gmira, L. Véron. Boundary singularities of solutions of some nonlinear elliptic equations // Duke Math. J. 1991. V. 64. № 2. P. 271-324.

[11] S.E. Kuznetsov. $\sigma$-moderate solutions of $L u=u^{\alpha}$ and fine trace on the boundary // C. R. Acad. Sci. Paris Sér. I Math. 1998. V. 326. № 10. P. 1189-1194.

[12] S.E. Kuznetsov. An upper bound for positive solutions of the equation $\Delta u=u^{\alpha}$, in preparation.

[13] J.-F. Le Gall. Les solutions positives de $\Delta u=u^{2}$ dans le disque unité // C. R. Acad. Sci. Paris Sér. I Math. 1993. V. 317. № 9. P. 873-878. 
[14] J.-F. Le Gall. A probabilistic Poisson representation for positive solutions of $\Delta u=u^{2}$ in a planar domain // Comm. Pure Appl. Math. 1997. V. 50. № 1. P. 69-103.

[15] M. Marcus, L. Véron. The boundary trace of positive solutions of semilinear elliptic equations: The subcritical case // Arch. Rational Mech. Anal. 1998. V. 144. № 3. P. 201-231.

[16] M. Marcus, L. Véron. The boundary trace of positive solutions of semilinear elliptic equations: The supercritical case // J. Math. Pures Appl. (9). 1998. V. 77. № 5. P. 481-524.

[17] M. Marcus, L. Véron. Capacitary estimates of solutions of a class of nonlinear elliptic equations // C. R. Math. Acad. Sci. Paris. 2003. V. 336. №11. P. 913-918.

[18] B. Mselati. Classification et représentation probabiliste des solutions positives de $\Delta u=u^{2}$ dans un domaine // Thése de Doctorat de l'Université Paris VI, 2002.

University, Ithaca, NY, USA

Поступила в редакцию

E-mail: ebd1@cornell.edu

09.09.2003 